Document downloaded from:

http://hdl.handle.net/10251/56036

This paper must be cited as:

Cordero Barbero, A.; Torregrosa Sánchez, JR.; Penkova Vassileva, M. (2013). New family of iterative methods with high order of convergence for solving nonlinear systems. En Numerical Analysis and Its Applications. Springer Verlag. 222-230. doi:10.1007/978-3-64241515-9_23.

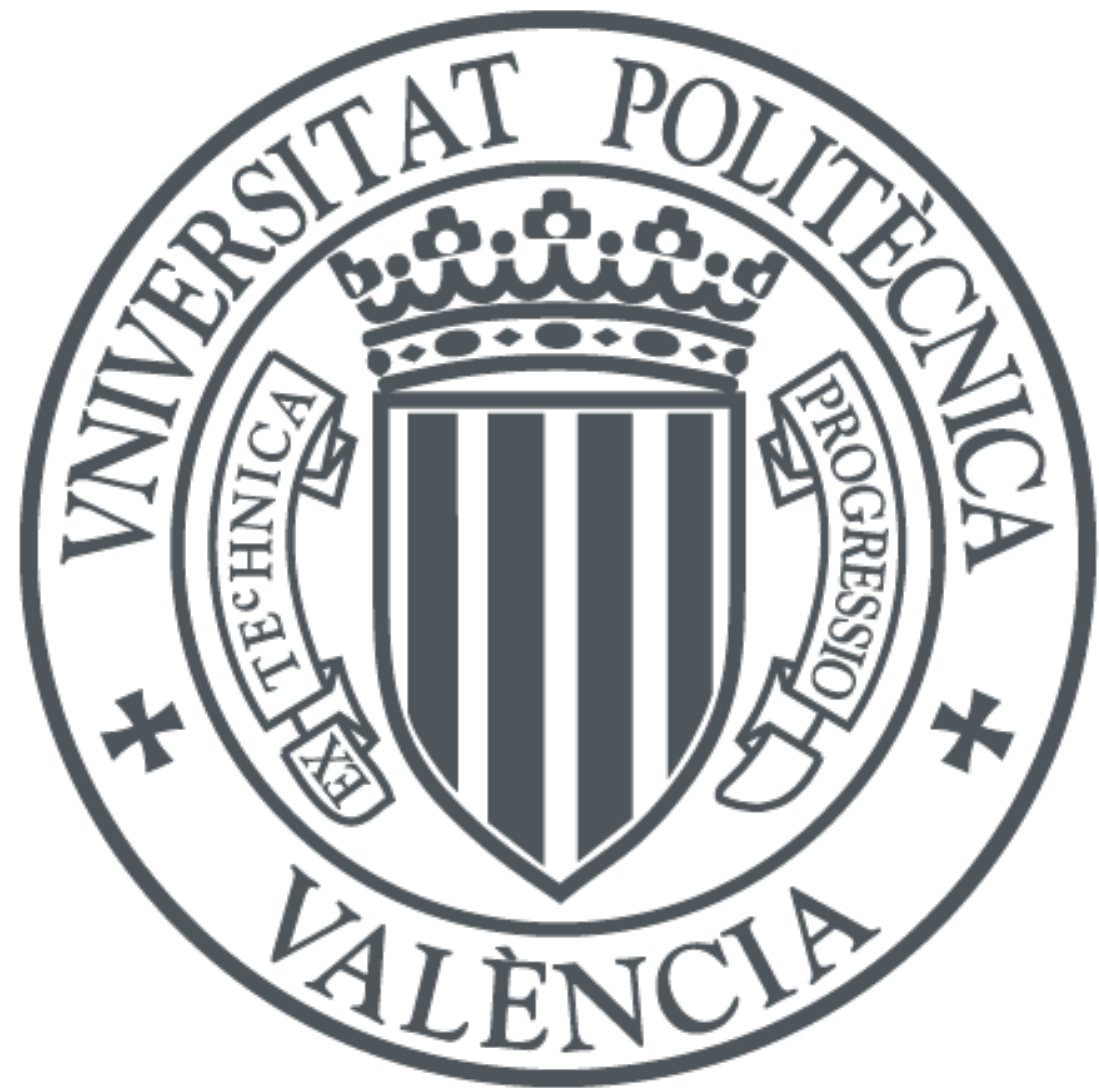

The final publication is available at

http://dx.doi.org/10.1007/978-3-642-41515-9_23

Copyright Springer Verlag

Additional Information 


\title{
New Family of Iterative Methods with High Order of Convergence for Solving Nonlinear Systems
}

\author{
Alicia Cordero $^{1}$, Juan R. Torregrosa ${ }^{1}$, and María P. Vassileva ${ }^{2}$ \\ 1 Instituto de Matemática Multidisciplinar, \\ Universitat Politècnica de València, \\ Camino de Vera, s/n 40022 Valencia, España, \\ acordero@mat.upv.es, jrtorre@mat.upv.es, \\ 2 Instituto Tecnológico de Santo Domingo (INTEC), \\ av. Los Próceres, Galá, Santo Domingo, \\ República Dominicana, \\ marip@intec.edu.do,
}

\begin{abstract}
In this paper we present and analyze a set of predictor-corrector iterative methods with increasing order of convergence, for solving systems of nonlinear equations. Our aim is to achieve high order of convergence with few Jacobian and/or functional evaluations. On the other hand, by applying the pseudocomposition technique on each proposed scheme we get to increase their order of convergence, obtaining new high-order and efficient methods. We use the classical efficiency index in order to compare the obtained schemes and make some numerical test.
\end{abstract}

Keywords: Nonlinear systems, Iterative methods, Jacobian matrix, Convergence order, Efficiency index

\section{Introduction}

Many relationships in nature are inherently nonlinear, which according to these effects are not in direct proportion to their cause. In fact, a large number of such real-world applications are reduce to solve nonlinear systems numerically. Approximating a solution $\xi$ of a nonlinear system $F(x)=0$, is a classical problem that appears in different branches of science and engineering.

Recently, for $n=1$, many robust and efficient methods have been proposed with high convergence order, but in most of cases the method cannot be extended for several variables. Few papers for the multidimensional case introduce methods with high order of convergence. The authors design in [1] a modified Newton-Jarrat scheme of sixth-order; in [6] a third-order method is presented for computing real and complex roots of nonlinear systems; Shin et al. compare in [8] Newton-Krylov methods and Newton-like schemes for solving big-sized nonlinear systems; in [2] a general procedure to design high-order methods for problems in several variables is presented.

The pseudocomposition technique (see [5]) consists of the following: we consider a method of order of convergence $p$ as a predictor, whose penultimate step is of order $q$, and then we use a corrector step based on the Gaussian quadrature. So, we obtain a family of iterative schemes whose order of convergence is $\min \{q+p, 3 q\}$. This is a general procedure to improve the order of convergence of known methods.

To analyze and compare the efficiency of the proposed methods we use the classic efficiency index $I=p^{1 / d}$ due to Ostrowski [7], where $p$ is the order of convergence, $d$ is the number of functional evaluations, per iteration.

In this paper, we present three new Newton-like schemes, of order of convergence four, six and eight, respectively. After the analysis of convergence of the new methods, we apply the pseudocomposition technique in order to get higher order procedures.

The convergence theorem in Section 2 can be demonstrated by means of the n-dimensional Taylor expansion of the functions involved. Let $F: D \subseteq R^{n} \longrightarrow R^{n}$ be sufficiently Frechet differentiable in $D$. By using the notation introduced in [1], the $q$ th derivative of $F$ at $u \in R^{n}, q \geq 1$, is the $q$-linear function $F^{(q)}(u): R^{n} \times \cdots \times$ $R^{n} \longrightarrow R^{n}$ such that $F^{(q)}(u)\left(v_{1}, \ldots, v_{q}\right) \in R^{n}$. It is easy to observe that

1. $F^{(q)}(u)\left(v_{1}, \ldots, v_{q-1}, \cdot\right) \in \mathcal{L}\left(R^{n}\right)$,

\footnotetext{
* This research was supported by Ministerio de Ciencia y Tecnología MTM2011-28636-C02-02 and by FONDOCYT 2011-1-B1-33, República Dominicana.
} 
2. $F^{(q)}(u)\left(v_{\sigma(1)}, \ldots, v_{\sigma(q)}\right)=F^{(q)}(u)\left(v_{1}, \ldots, v_{q}\right)$, for all permutation $\sigma$ of $\{1,2 \ldots, q\}$.

So, in the following we will denote:

(a) $F^{(q)}(u)\left(v_{1}, \ldots, v_{q}\right)=F^{(q)}(u) v_{1} \ldots v_{q}$,

(b) $F^{(q)}(u) v^{q-1} F^{(p)} v^{p}=F^{(q)}(u) F^{(p)}(u) v^{q+p-1}$.

It is well known that, for $\xi+h \in R^{n}$ lying in a neighborhood of a solution $\xi$ of the nonlinear system $F(x)=0$, Taylor's expansion can be applied (assuming that the Jacobian matrix $F^{\prime}(\xi)$ is nonsingular), and

$$
F(\xi+h)=F^{\prime}(\xi)\left[h+\sum_{q=2}^{p-1} C_{q} h^{q}\right]+O\left[h^{p}\right],
$$

where $C_{q}=(1 / q !)\left[F^{\prime}(\xi)\right]^{-1} F^{(q)}(\xi), q \geq 2$. We observe that $C_{q} h^{q} \in R^{n}$ since $F^{(q)}(\xi) \in \mathcal{L}\left(R^{n} \times \cdots \times R^{n}, R^{n}\right)$ and $\left[F^{\prime}(\xi)\right]^{-1} \in \mathcal{L}\left(R^{n}\right)$.

In addition, we can express the Jacobian matrix of $F, F^{\prime}$, as

$$
F^{\prime}(\xi+h)=F^{\prime}(\xi)\left[I+\sum_{q=2}^{p-1} q C_{q} h^{q-1}\right]+O\left[h^{p}\right],
$$

where $I$ is the identity matrix. Therefore, $q C_{q} h^{q-1} \in \mathcal{L}\left(R^{n}\right)$. From (2), we obtain

$$
\left[F^{\prime}(\xi+h)\right]^{-1}=\left[I+X_{2} h+X_{3} h^{2}+X_{4} h^{3}+\cdots\right]\left[F^{\prime}(\xi)\right]^{-1}+O\left[h^{p}\right],
$$

where $X_{2}=-2 C_{2}, X_{3}=4 C_{2}^{2}-3 C_{3}, \ldots$

We denote $e_{k}=x^{(k)}-\xi$ the error in the $k$ th iteration. The equation $e_{(k+1)}=L e_{k}{ }^{p}+O\left[e_{k}{ }^{p+1}\right]$, where $L$ is a $p$-linear function $L \in \mathcal{L}\left(R^{n} \times \cdots \times R^{n}, R^{n}\right)$, is called the error equation and $p$ is the order of convergence.

The rest of the paper is organized as follows: in the next section, we present the new methods of order four, six and eight, respectively. Moreover, the convergence order is increased when the pseudocomposition technique is applied. Section 3 is devoted to the comparison of the different methods by means of several numerical tests.

\section{Proposed high-order methods}

Let us introduce now a new Jarratt-type scheme of five steps which we will denote as M8. It can be proved that its first three steps are a fourth-order scheme, denoted by M4, and its four first steps become a sixth-order method that will be denoted by M6. The coefficients involved have been obtained optimizing the order the convergence and the whole scheme requires three functional evaluations of $F$ and two of $F^{\prime}$ to attain eighthorder of convergence. Let us also note that the linear systems to be solved in first, second and last step have the same matrix and also have the third and fourth steps, so the number of operations involved is not as high as it can seem.

Theorem 1. Let $F: \Omega \subseteq R^{n} \rightarrow R^{n}$ be sufficiently differentiable in a neighborhood of $\xi \in \Omega$ which is a solution of the nonlinear system $F(x)=0$. We suppose that $F^{\prime}(x)$ is continuous and nonsingular at $\xi$ and $x^{(0)}$ close enough to the solution. Then, the sequence $\left\{x^{(k)}\right\}_{k \geq 0}$ obtained by

$$
\begin{aligned}
y^{(k)} & =x^{(k)}-\frac{2}{3}\left[F^{\prime}\left(x^{(k)}\right)\right]^{-1} F\left(x^{(k)}\right), \\
z^{(k)} & =y^{(k)}+\frac{1}{6}\left[F^{\prime}\left(x^{(k)}\right)\right]^{-1} F\left(x^{(k)}\right), \\
u^{(k)} & =z^{(k)}+\left[F^{\prime}\left(x^{(k)}\right)-3 F^{\prime}\left(y^{(k)}\right)\right]^{-1} F\left(x^{(k)}\right), \\
v^{(k)} & =z^{(k)}+\left[F^{\prime}\left(x^{(k)}\right)-3 F^{\prime}\left(y^{(k)}\right)\right]^{-1}\left[F\left(x^{(k)}\right)+2 F\left(u^{(k)}\right)\right], \\
x^{(k+1)} & =v^{(k)}-\frac{1}{2}\left[F^{\prime}\left(x^{(k)}\right)\right]^{-1}\left[5 F^{\prime}\left(x^{(k)}\right)-3 F^{\prime}\left(y^{(k)}\right)\right]\left[F^{\prime}\left(x^{(k)}\right)\right]^{-1} F\left(v^{(k)}\right),
\end{aligned}
$$

converges to $\xi$ with order of convergence eight. The error equation is:

$$
e_{k+1}=\left(C_{2}^{2}-\frac{1}{2} C_{3}\right)\left(2 C_{2}^{3}+2 C_{3} C_{2}-2 C_{2} C_{3}-\frac{20}{9} C_{4}\right) e_{k}^{8}+O\left[e_{k}^{9}\right] .
$$


By applying the next result, it is known (see [5]) that, the pseudocomposition technique allows us to design methods with higher order of convergence.

Theorem 2. [5] Let $F: \Omega \subseteq R^{n} \rightarrow R^{n}$ be differentiable enough in $\Omega$ and $\xi \in \Omega$ a solution of the nonlinear system $F(x)=0$. We suppose that $F^{\prime}(x)$ is continuous and nonsingular at $\xi$. Let $y^{(k)}$ and $z^{(k)}$ be the penultimate and final steps of orders $q$ and $p$, respectively, of a certain iterative method. Taking this scheme as a predictor we get a new approximation $x^{(k+1)}$ of $\xi$ given by

$$
x^{(k+1)}=y^{(k)}-2\left[\sum_{i=1}^{m} \omega_{i} F^{\prime}\left(\eta_{i}^{(k)}\right)\right]^{-1} F\left(y^{(k)}\right),
$$

where $\eta_{i}^{(k)}=\frac{1}{2}\left[\left(1+\tau_{i}\right) z^{(k)}+\left(1-\tau_{i}\right) y^{(k)}\right]$ and $\tau_{i}, \omega_{i} i=1, \ldots, m$ are the nodes and weights of the orthogonal polynomial corresponding to the Gaussian quadrature used. Then,

1. the obtained set of families will have an order of convergence at least q;

2. if $\sigma=2$ is satisfied, then the order of convergence will be at least $2 q$;

3. if, also, $\sigma_{1}=0$ the order of convergence will be $\min \{p+q, 3 q\}$;

where $\sum_{i=1}^{n} \omega_{i}=\sigma$ and $\sum_{i=1}^{n} \frac{\omega_{i} \tau_{i}^{j}}{\sigma}=\sigma_{j} \quad$ with $\quad j=1,2$.

Each of the families obtained will consist of subfamilies that are determined by the orthogonal polynomial corresponding to the Gaussian quadrature used. Furthermore, in these subfamilies it can be obtained methods using different number of nodes corresponding to the orthogonal polynomial used (see Table 1). According to the proof of Theorem 2 the order of convergence of the obtained methods does not depend on the number of nodes used.

\begin{tabular}{|c|c|c|c|c|c|c|c|c|}
\hline \hline & \multicolumn{7}{|c|}{ Quadratures } \\
\hline Number of nodes & Chebyshev & \multicolumn{3}{|c|}{ Legendre } & Lobatto & Radau \\
\hline & $\sigma$ & $\sigma_{1}$ & $\sigma$ & $\sigma_{1}$ & $\sigma$ & $\sigma_{1}$ & $\sigma$ & $\sigma_{1}$ \\
\hline \hline 1 & $\pi$ & 0 & 2 & 0 & 2 & 0 & 2 & -1 \\
\hline 2 & $\pi$ & 0 & 2 & 0 & 2 & 0 & 2 & 0 \\
\hline 3 & $\pi$ & 0 & 2 & 0 & 2 & 0 & 2 & 0 \\
\hline
\end{tabular}

Table 1: Quadratures used

Let us note that these methods, obtained by means of Gaussian quadratures, seem to be known interpolation quadrature schemes such as midpoint, trapezoidal or Simpson's method (see [4]). It is only a similitude, as they are not applied on the last iteration $x^{(k)}$, and the last step of the predictor $z^{(k)}$, but on the two last steps of the predictor. In the following, we will use a midpoint-like as a corrector step, which corresponds to a GaussLegendre quadrature with one node; for this scheme the order of convergence will be at least $\min \{q+p, 3 q\}$, by applying Theorem 2 .

The pseudocomposition can be applied on the proposed scheme M8 with iterative expression (4), but also on M6. By pseudocomposing on M6 and M8 there can be obtained two procedures of order of convergence 10 and 14 (denoted by PsM10 and PsM14), respectively. Let us note that it is also possible to pseudocompose on M4, but the resulting scheme would be of third order of convergence, which is worst than the original M4, so it will not be considered.

Following the notation used in (4), the last step of PsM10 is

$$
x^{(k+1)}=u^{(k)}-2\left[F^{\prime}\left(\frac{v^{(k)}+u^{(k)}}{2}\right)\right]^{-1} F\left(u^{(k)}\right),
$$


and the last three steps of psM14 can be expressed as

$$
\begin{aligned}
v^{(k)} & =z^{(k)}+\left[F^{\prime}\left(x^{(k)}\right)-3 F^{\prime}\left(y^{(k)}\right)\right]^{-1}\left[F\left(x^{(k)}\right)+2 F\left(u^{(k)}\right)\right], \\
w^{(k+1)} & =v^{(k)}-\frac{1}{2}\left[F^{\prime}\left(x^{(k)}\right)\right]^{-1}\left[5 F^{\prime}\left(x^{(k)}\right)-3 F^{\prime}\left(y^{(k)}\right)\right]\left[F^{\prime}\left(x^{(k)}\right)\right]^{-1} F\left(v^{(k)}\right), \\
x^{(k+1)} & =v^{(k)}-2\left[F^{\prime}\left(\frac{w^{(k)}+v^{(k)}}{2}\right)\right]^{-1} F\left(v^{(k)}\right) .
\end{aligned}
$$

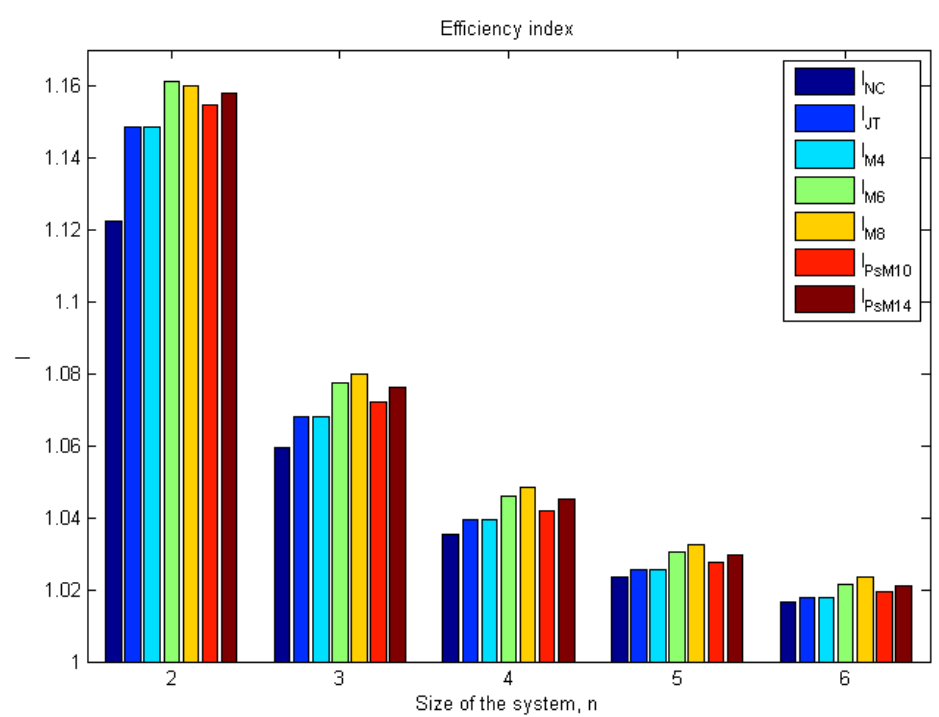

Fig. 1: Efficiency index of the different methods for different sizes of the system

If we analyze the efficiency indices (see Figure 1, we deduce the following conclusions: the new methods M4, M6 and M8 (and also the pseudocomposed PsM10 and PsM14) improve Newton and Jarratt's schemes (in fact, the indices of M4 and Jarratt's are equal). Indeed, for $n \geq 3$ the best index is that of M8. Nevertheless, none of the pseudocomposed methods improve the efficiency index of their original partners.

\section{Numerical results}

In this section, we test the developed methods to illustrate its effectiveness compared with other methods. Numerical computations have been performed in MATLAB R2011a by using variable-precision arithmetic, which uses floating-point representation of 2000 decimal digits of mantissa. The computer specifications are: Intel(R) Core(TM) i5-2500 CPU @ 3.30GHz with 16.00GB of RAM. Each iteration is obtained from the former by means of an iterative expression $x^{(k+1)}=x^{(k)}-A^{-1} b$, where $x^{(k)} \in \mathbb{R}^{n}, A$ is a real matrix $n \times n$ and $b \in \mathbb{R}^{n}$. The matrix $A$ and vector $b$ are different according to the method used, but in any case, we use to calculate inverse $-A^{-1} b$ the solution of the linear system $A y=b$, with Gaussian elimination with partial pivoting. The stopping criterion used is $\left\|x^{(k+1)}-x^{(k)}\right\|<10^{-200}$ or $\left\|F\left(x^{(k)}\right)\right\|<10^{-200}$.

Firstly, let us consider the following nonlinear systems of different sizes:

1. $F_{1}=\left(f_{1}(x), f_{2}(x), \ldots, f_{n}(x)\right)$, where $x=\left(x_{1}, x_{2}, \ldots, x_{n}\right)^{T}$ and $f_{i}: \mathbb{R}^{n} \rightarrow \mathbb{R}, i=1,2, \ldots, n$, such that

$$
\begin{aligned}
f_{i}(x) & =x_{i} x_{i+1}-1, i=1,2, \ldots, n-1 \\
f_{n}(x) & =x_{n} x_{1}-1 .
\end{aligned}
$$

When $n$ is odd, the exact zeros of $F_{1}(x)$ are: $\xi_{1}=(1,1, \ldots, 1)^{T}$ and $\xi_{2}=(-1,-1, \ldots,-1)^{T}$. 
2. $F_{2}\left(x_{1}, x_{2}\right)=\left(x_{1}^{2}-x_{1}-x_{2}^{2}-1,-\sin \left(x_{1}\right)+x_{2}\right)$ and the solutions are $\xi_{1} \approx(-0.845257,-0.748141)^{T}$ and $\xi_{2} \approx(1.952913,0.927877)^{T}$

3. $F_{3}\left(x_{1}, x_{2}\right)=\left(x_{1}^{2}+x_{2}^{2}-4,-\exp \left(x_{1}\right)+x_{2}-1\right)$, being the solutions $\xi_{1} \approx(1.004168,-1.729637)^{T}$ and $\xi_{2} \approx(-1.816264,0.837368)^{T}$.

4. $F_{4}\left(x_{1}, x_{2}, x_{3}\right)=\left(x_{1}^{2}+x_{2}^{2}+x_{3}^{2}-9, x_{1} x_{2} x_{3}-1, x_{1}+x_{2}-x_{3}^{2}\right)$ with three roots $\xi_{1} \approx(2.14025,-2.09029,-0.223525)^{T}$, $\xi_{2} \approx(2.491376,0.242746,1.653518)^{T}$ and $\xi_{1} \approx(0.242746,2.491376,1.653518)^{T}$.

Table 2 presents results showing the following information: the different iterative methods employed (Newton (NC), Jarratt (JT), the new methods M4, M6 and M8 and the pseudocomposed PsM10 and PsM14), the number of iterations Iter needed to converge to the solution Sol, the value of the stopping factors at the last step and the computational order of convergence $\rho$ (see [3]) approximated by the formula:

$$
\rho \approx \frac{\ln \left(\left\|x^{(k+1)}-x^{(k)}\right\|\right) /\left(\left\|x^{(k)}-x^{(k-1)}\right\|\right)}{\ln \left(\left\|x^{(k)}-x^{(k-1)}\right\|\right) /\left(\left\|x^{(k-1)}-x^{(k-2)}\right\|\right)} .
$$

The value of $\rho$ which appears in Table 2 is the last coordinate of the vector $\rho$ when the variation between their coordinates is small. Also the elapsed time, in seconds, appears in Table 2, being the mean execution time for 100 performances of the method (the command cputime of Matlab has been used).

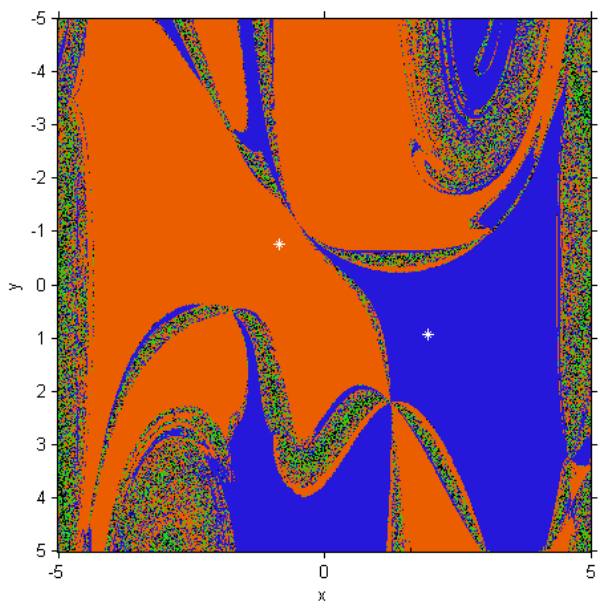

(a) $M 6$

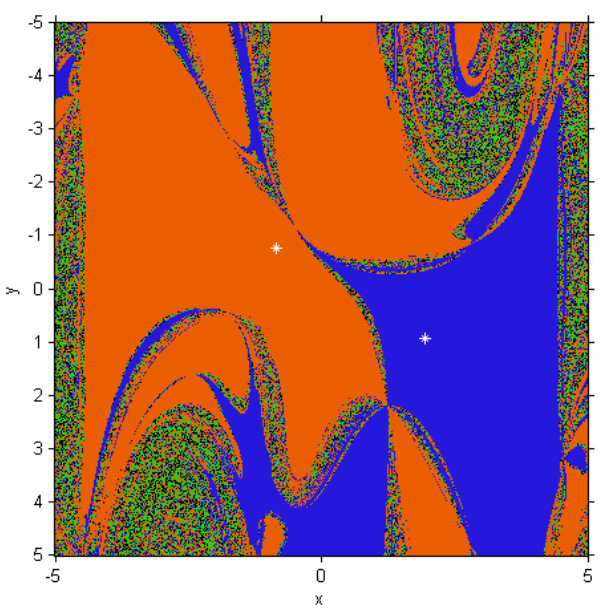

(b) $P s M 10$

Fig. 2

We observe from Table 2 that, not only the order of convergence and the number of new functional evaluations and operations is important in order to obtain new efficient iterative methods to solve nonlinear systems of equations. A key factor is the range of applicability of the methods. Although they are slower than the original methods when the initial estimation is quite good, when we are far from the solution or inside a region of instability, the original schemes do not converge or do it more slowly, the corresponding pseudocomposed procedures usually still converge or do it faster.

The advantage of pseudocomposition can be observed in Figures 2a, 2b (methods M6 and PsM10) and 3a, 3b (methods M8 and PsM14) where the dynamical plane on $R^{2}$ is represented: let us consider a system of two equations and two unknowns (the case $F_{2}(x)=0$ is showed), for any initial estimation in $\mathbb{R}^{2}$ represented by its position in the plane, a different color (blue or orange, as there exist only two solutions) is used for the different solutions found (marked by a white point in the figure). Black color represents an initial point in which the method converges to infinity, and the green one means that no convergence is found (usually because any linear system cannot be solved). It is clear that when many initial estimations tend to infinity (see Figure 3a), the pseudocomposition "cleans" the dynamical plane, making the method more stable as it can find one of the solutions by using starting points that do not allow convergence with the original scheme (see Figure $2 \mathrm{~b}$ ). 


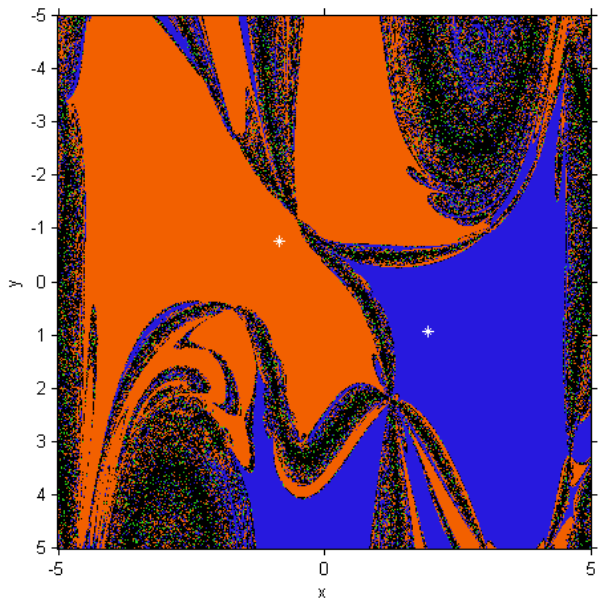

(a) $M 8$

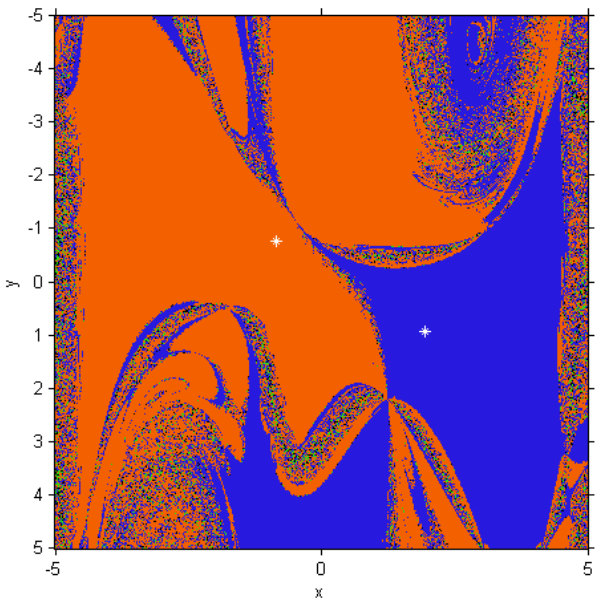

(b) PsM14

Fig. 3: Real dynamical planes for system $(b)$ and methods M8 and PsM14

We conclude that the presented schemes M4, M6 and M8 show to be excellent, in terms of order of convergence and efficiency, but also that the pseudocomposition technique achieves to transform them in competent and more robust new schemes.

\section{References}

1. A. Cordero, J.L. Hueso, E. Martínez, J.R. Torregrosa:A modified Newton-Jarratt's composition. Numer. Algor. 55, 87-99 (2010)

2. A. Cordero, J.L. Hueso, E. Martínez, J.R. Torregrosa: Efficient high-order methods based on golden ratio for nonlinear systems. Applied Mathematics and Computation 217(9), 4548-4556 (2011)

3. A. Cordero, J.R. Torregrosa: Variants of Newton's Method using fifth-order quadrature formulas. Applied Mathematics and Computation 190, 686-698 (2007)

4. A. Cordero, J.R. Torregrosa: On interpolation variants of Newton's method for functions of several variables. Journal of Computational and Applied Mathematics 234, 34-43 (2010)

5. A. Cordero, J.R. Torregrosa, M.P. Vassileva: Pseudocomposition: a technique to design predictor-corrector methods for systms of nonlinear equtaions. Applied Mathematics and Computation 218(23), 11496-11504 (2012)

6. M. Nikkhah-Bahrami, R. Oftadeh: An effective iterative method for computing real and complex roots of systems of nonlinear equations. Applied Mathematics and Computation 215, 1813-1820 (2009)

7. A. M. Ostrowski: Solutions of equations and systems of equations. Academic Press, New York-London 1966

8. Byeong-Chun Shin, M.T. Darvishi, Chang-Hyun Kim: A comparison of the Newton-Krylov method with high order Newton-like methods to solve nonlinear systems. Applied Mathematics and Computation 217, 3190-3198 (2010) 


\begin{tabular}{|c|c|c|c|c|c|c|c|}
\hline Function & |Method & Iter & $\mid$ Sol|| & $\mid \overline{\left|x^{(k)}-x^{(k-1)}\right| \mid}$ & ||$F\left(x^{(k)} \| \mid\right.$ & 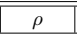 & $\mid$ e-time (sec) \\
\hline$F_{1}$ & $\overline{\mathrm{NC}}$ & 8 & \begin{tabular}{l|l}
$\xi_{1}$ \\
$\xi_{1}$
\end{tabular} & $\overline{~ 1.43 \mathrm{e}-121}$ & \begin{tabular}{|l|}
$2.06 \mathrm{e}-243$ \\
\end{tabular} & 2.0000 & 8.6407 \\
\hline \multirow[t]{6}{*}{$x^{(0)}=(0.8, \ldots, 0.8) n=99$} & JT & 4 & $\xi_{1}$ & $1.69 \mathrm{e}-60$ & \begin{tabular}{|l|}
$2.06 \mathrm{e}-243$ \\
\end{tabular} & 4.0000 & 3.9347 \\
\hline & M4 & 4 & $\xi_{1}$ & $1.69 \mathrm{e}-60$ & \begin{tabular}{|l|}
$2.06 \mathrm{e}-243$ \\
\end{tabular} & 4.0000 & 3.7813 \\
\hline & M6 & 4 & $\xi_{1}$ & $6.94 \mathrm{e}-193$ & $4.33 \mathrm{e}-1160$ & \begin{tabular}{|l|}
6.0000 \\
\end{tabular} & 5.3911 \\
\hline & M8 & 3 & $\xi_{1}$ & $9.40 \mathrm{e}-50$ & $3.51 \mathrm{e}-4011$ & \begin{tabular}{|l|}
8.0913 \\
\end{tabular} & 5.0065 \\
\hline & \begin{tabular}{|l|} 
PsM10 \\
\end{tabular} & 3 & \begin{tabular}{|l|}
$\xi_{1}$ \\
\end{tabular} & $1.28 \mathrm{e}-91$ & \begin{tabular}{|c|}
$9.54 \mathrm{e}-921$ \\
\end{tabular} & 10.0545 & 4.9061 \\
\hline & PsM14 & 3 & $\xi_{1}$ & $4.65 \mathrm{e}-164$ & 0 & 14.0702 & 6.1018 \\
\hline$F_{1}$ & NC & 17 & $\xi_{1}$ & $\begin{array}{l}3.37 \mathrm{e}-340 \\
\end{array}$ & \begin{tabular}{|l|l|}
$1.14 \mathrm{e}-340$ \\
\end{tabular} & $\overline{-1}$ & 9.2128 \\
\hline \multirow[t]{6}{*}{$x^{(0)}=(0.0015, \ldots, 0.0015) n=99$} & JT & 9 & $\xi_{1}$ & $8.18 \mathrm{e}-085$ & \begin{tabular}{|l|l|}
$1.14 \mathrm{e}-340$ \\
\end{tabular} & 4.0000 & 10.1416 \\
\hline & M4 & 9 & $\xi_{1}$ & $8.18 \mathrm{e}-085$ & \begin{tabular}{|l|l|}
$1.14 \mathrm{e}-340$ \\
\end{tabular} & 4.0000 & 10.9104 \\
\hline & M6 & 7 & $\xi_{1}$ & $1.40 \mathrm{e}-035$ & \begin{tabular}{|l|}
$9.46 \mathrm{e}-216$ \\
\end{tabular} & - & 12.3266 \\
\hline & M8 & 19 & $\xi_{1}$ & $9.50 \mathrm{e}-030$ & \begin{tabular}{|l|}
$1.29 \mathrm{e}-240$ \\
\end{tabular} & - & 59.4832 \\
\hline & \begin{tabular}{|l|} 
PsM10 \\
\end{tabular} & 6 & $\xi_{1}$ & $3.02 \mathrm{e}-102$ & $5.23 \mathrm{e}-1027$ & - & 17.9957 \\
\hline & PsM14 & 5 & \begin{tabular}{|l|}
$\xi_{1}$ \\
\end{tabular} & $1.84 \mathrm{e}-162$ & 0 & - & 22.6130 \\
\hline$\overline{F_{2}}$ & NC & 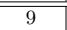 & $\xi_{1}$ & 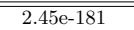 & \begin{tabular}{|l|}
$5.92 \mathrm{e}-362$ \\
\end{tabular} & \begin{tabular}{|l|l|}
2.0148 \\
\end{tabular} & 0.2395 \\
\hline \multirow[t]{6}{*}{$x^{(0)}=(-0.5,-0.5)$} & JT & 5 & $\xi_{1}$ & $9.48 \mathrm{e}-189$ & $8.13 \mathrm{e}-754$ & \begin{tabular}{|l|}
4.0279 \\
\end{tabular} & 0.3250 \\
\hline & M4 & 5 & $\xi_{1}$ & $9.48 \mathrm{e}-189$ & $8.13 \mathrm{e}-754$ & \begin{tabular}{|l|}
4.0279 \\
\end{tabular} & 0.1841 \\
\hline & M6 & 4 & $\xi_{1}$ & $1.34 \mathrm{e}-146$ & \begin{tabular}{|l|}
$2.14 \mathrm{e}-878$ \\
\end{tabular} & 5.9048 & 0.2744 \\
\hline & M8 & 3 & \begin{tabular}{|c|}
1 \\
$\xi_{1}$
\end{tabular} & $1.90 \mathrm{e}-038$ & \begin{tabular}{|l|}
$1.23 \mathrm{e}-302$ \\
\end{tabular} & 7.8530 & 0.3718 \\
\hline & PsM10 & 3 & \begin{tabular}{|l|}
$\xi_{1}$ \\
\end{tabular} & $6.72 \mathrm{e}-72$ & \begin{tabular}{|l|}
$2.68 \mathrm{e}-714$ \\
\end{tabular} & \begin{tabular}{|l|}
9.9092 \\
\end{tabular} & 0.4674 \\
\hline & PsM14 & 3 & \begin{tabular}{|l|l|}
$\xi_{1}$ \\
\end{tabular} & $2.13 \mathrm{e}-122$ & $\mid 1.95 \mathrm{e}-1706$ & 13.9829 & 0.3187 \\
\hline$F_{2}$ & NC & 13 & \begin{tabular}{|l|l|}
$\xi_{1}$ \\
\end{tabular} & $2.20 \mathrm{e}-182$ & \begin{tabular}{|l|}
$2.73 \mathrm{e}-374$ \\
\end{tabular} & \begin{tabular}{|l|l|}
1.9917 \\
\end{tabular} & 0.3713 \\
\hline \multirow{6}{*}{$x^{(0)}=(-5,-3)$} & JT & 7 & $\xi_{1}$ & $2.10 \mathrm{e}-179$ & \begin{tabular}{|l|l|}
$4.51 \mathrm{e}-716$ \\
\end{tabular} & 3.9925 & 0.4001 \\
\hline & M4 & 7 & $\xi_{1}$ & $2.10 \mathrm{e}-179$ & \begin{tabular}{|l|}
$4.51 \mathrm{e}-716$ \\
\end{tabular} & 3.9925 & 0.7535 \\
\hline & M6 & 8 & \begin{tabular}{|l|}
$\xi_{1}$ \\
\end{tabular} & $2.55 \mathrm{e}-036$ & \begin{tabular}{|l|}
$5.81 \mathrm{e}-216$ \\
\end{tabular} & - & 0.9382 \\
\hline & M8 & $>5000$ & & & & & \\
\hline & PsM10 & 4 & $\xi_{1}$ & $2.59 \mathrm{e}-021$ & \begin{tabular}{|l|}
$3.51 \mathrm{e}-208$ \\
\end{tabular} & - & 0.4363 \\
\hline & PsM14 & 29 & \begin{tabular}{|l|}
$\xi_{2}$ \\
\end{tabular} & $9.45 \mathrm{e}-020$ & $5.05 \mathrm{e}-273$ & - & 7.8090 \\
\hline$F_{3}$ & $\overline{\mathrm{NC}}$ & 10 & \begin{tabular}{l|l}
$\xi_{1}$ \\
\end{tabular} & $\bar{~} 1.65 \mathrm{e}-190$ & \begin{tabular}{|l|l|}
$4.61 \mathrm{e}-380$ \\
\end{tabular} & 2.0000 & 1.4675 \\
\hline \multirow[t]{6}{*}{$x^{(0)}=(2,-3)$} & JT & 5 & $\xi_{1}$ & $8.03 \mathrm{e}-113$ & \begin{tabular}{|l}
$7.59 \mathrm{e}-450$ \\
\end{tabular} & 3.9995 & 0.3151 \\
\hline & M4 & 5 & $\xi_{1}$ & $8.03 \mathrm{e}-113$ & \begin{tabular}{|l|l|}
$7.59 \mathrm{e}-450$ \\
\end{tabular} & 3.9995 & 0.3034 \\
\hline & M6 & 4 & $\xi_{1}$ & $1.25 \mathrm{e}-082$ & \begin{tabular}{|l|}
$2.83 \mathrm{e}-493$ \\
\end{tabular} & 6.0015 & 0.3696 \\
\hline & M8 & 4 & $\xi_{1}$ & $1.54 \mathrm{e}-162$ & $3.16 \mathrm{e}-1296$ & 7.9993 & 0.4463 \\
\hline & PsM10 & 3 & $\xi_{1}$ & $5.59 \mathrm{e}-044$ & \begin{tabular}{|l|}
$1.40 \mathrm{e}-436$ \\
\end{tabular} & 9.4708 & 0.4682 \\
\hline & PsM14 & 3 & $\xi_{1}$ & $3.46 \mathrm{e}-068$ & \begin{tabular}{|l|}
$3.45 \mathrm{e}-948$ \\
\end{tabular} & 13.1659 & 0.5925 \\
\hline$\overline{F_{3}}$ & "NC & "35 & $\xi_{1}$ & $3.71 \mathrm{e}-177$ & \begin{tabular}{|l|}
$2.33 \mathrm{e}-253$ \\
\end{tabular} & "- & 1.4828 \\
\hline \multirow[t]{6}{*}{$x^{(0)}=(0.2,0.1)$} & JT & 11 & $\xi_{1}$ & $3.29 \mathrm{e}-143$ & \begin{tabular}{|l|}
$1.67 \mathrm{e}-574$ \\
\end{tabular} & - & 0.7781 \\
\hline & M4 & 11 & \begin{tabular}{|l|}
$\xi_{1}$ \\
\end{tabular} & $3.29 \mathrm{e}-143$ & \begin{tabular}{|l|}
$1.67 \mathrm{e}-574$ \\
\end{tabular} & - & 0.7535 \\
\hline & M6 & 9 & \begin{tabular}{|l|}
$\xi_{1}$ \\
\end{tabular} & $1.31 \mathrm{e}-064$ & \begin{tabular}{|l|}
$3.61 \mathrm{e}-385$ \\
\end{tabular} & - & 0.8001 \\
\hline & M8 & n.c. & $\xi_{1}$ & & & & \\
\hline & PsM10 & 5 & $\xi_{1}$ & $6.85 \mathrm{e}-156$ & $1.06 \mathrm{e}-1555$ & - & 0.6352 \\
\hline & PsM14 & 8 & \begin{tabular}{|l|}
$\xi_{2}$ \\
\end{tabular} & $7.87 \mathrm{e}-155$ & 0 & - & 1.1870 \\
\hline$\overline{F_{4}}$ & $\overline{\mathrm{NC}}$ & $\overline{10}$ & $\xi_{1}$ & $\bar{~} 1.03 \mathrm{e}-135$ & \begin{tabular}{|l|}
$1.55 \mathrm{e}-270$ \\
\end{tabular} & 1.9995 & 2.3263 \\
\hline \multirow[t]{6}{*}{$x^{(0)}=(1,-1.5,-0.5)$} & JT & 5 & $\xi_{1}$ & $9.94 \mathrm{e}-073$ & $2.09 \mathrm{e}-289$ & 4.0066 & 0.5296 \\
\hline & M4 & 5 & $\xi_{1}$ & $9.94 \mathrm{e}-073$ & \begin{tabular}{|l|}
$2.09 \mathrm{e}-289$ \\
\end{tabular} & 4.0066 & 0.6340 \\
\hline & M6 & 4 & $\xi_{1}$ & $9.31 \mathrm{e}-057$ & \begin{tabular}{|l|}
$4.86 \mathrm{e}-338$ \\
\end{tabular} & 5.9750 & 0.7443 \\
\hline & M8 & 4 & $\xi_{1}$ & $4.43 \mathrm{e}-046$ & \begin{tabular}{|l|}
$1.08 \mathrm{e}-364$ \\
\end{tabular} & - & 0.8282 \\
\hline & PsM10 & 3 & $\xi_{1}$ & $1.43 \mathrm{e}-031$ & \begin{tabular}{|l|}
$1.04 \mathrm{e}-311$ \\
\end{tabular} & 9.6674 & 0.8100 \\
\hline & PsM14 & 3 & \begin{tabular}{|l|}
$\xi_{1}$ \\
\end{tabular} & $1.91 \mathrm{e}-033$ & \begin{tabular}{|l|}
$4.05 \mathrm{e}-462$ \\
\end{tabular} & 13.9954 & 1.0465 \\
\hline$\overline{F F_{4}}$ & 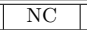 & 12 & $\xi_{1}$ & $\overline{1.08 \mathrm{e}-192}$ & \begin{tabular}{|l|}
$1.55 \mathrm{e}-384$ \\
\end{tabular} & \begin{tabular}{|l|}
1.9996 \\
\end{tabular} & 2.7271 \\
\hline \multirow[t]{6}{*}{$x^{(0)}=(7,-5,-5)$} & JT & 6 & \begin{tabular}{|l|}
$\xi_{1}$ \\
\end{tabular} & $2.31 \mathrm{e}-103$ & \begin{tabular}{|l|}
$7.97 \mathrm{e}-412$ \\
\end{tabular} & 4.0090 & 0.7761 \\
\hline & M4 & 6 & $\xi_{1}$ & $2.31 \mathrm{e}-103$ & \begin{tabular}{|l|}
$7.97 \mathrm{e}-412$ \\
\end{tabular} & 4.0090 & 1.0301 \\
\hline & M6 & 5 & $\xi_{1}$ & $2.99 \mathrm{e}-086$ & \begin{tabular}{|l|}
$4.69 \mathrm{e}-515$ \\
\end{tabular} & - & 1.0090 \\
\hline & M8 & 15 & \begin{tabular}{|l|}
$\xi_{3}$ \\
\end{tabular} & $1.77 \mathrm{e}-071$ & $1.48 \mathrm{e}-568$ & - & 3.4007 \\
\hline & PsM10 & 4 & \begin{tabular}{|l|}
$\xi_{1}$ \\
\end{tabular} & $6.86 \mathrm{e}-067$ & \begin{tabular}{|l|}
$1.25 \mathrm{e}-666$ \\
\end{tabular} & - & 1.0245 \\
\hline & PsM14 & 7 & \begin{tabular}{|l|}
$\xi_{2}$ \\
\end{tabular} & $1.09 \mathrm{e}-130$ & $9.15 \mathrm{e}-1825$ & - & 1.8179 \\
\hline
\end{tabular}

Table 2: Numerical results for functions $F_{1}$ to $F_{4}$ 